\title{
INVENTÁRIO ARBÓREO-URBANO DO MUNICÍPIO DE SALTO DE PIRAPORA, SP
}

\author{
Daniele Porto Benatti ${ }^{2}$, Kelly Cristina Tonello ${ }^{3}$, Francisco Carlos Adriano Júnior ${ }^{2}$, José Mauro Santana
} da Silva ${ }^{3}$, Ivanka Rosada de Oliveira ${ }^{2}$, Elce N. Rolim ${ }^{2}$ e Deborah L. Ferraz ${ }^{2}$

\begin{abstract}
RESUMO - Este estudo teve por objetivo realizar o inventário arbóreo-urbano em 24 bairros do Município de Salto de Pirapora, SP, por meio da avaliação quali-quantitativa. Para tanto, foram obtidas informações relacionadas à: espécie arbórea, situação da copa e do tronco, orientação do tronco, fitossanidade, interceptação das raízes no passeio, necessidade de tratos silviculturais, altura total e altura da primeira bifurcação, assim como características do espaço viário. Foram registrados 868 indivíduos, dos quais 679 foram catalogados em 71 espécies arbóreas. A espécie de maior frequência foi Caesalpinia pluviosa, representando 13,6\% do total, seguida de Ficus benjamin (10,4\%), Lagerstroemia indica $(5,0 \%)$ e Terminalia catappa $(4,8 \%)$. Quanto à condição do tronco, $68,8 \%$ dos indivíduos arbóreos não apresentaram problemas; $85,7 \%$ das árvores observadas possuíam orientação simpodial adequada; poucas árvores apresentavam danos relacionados à fitossanidade que poderiam interferir em sua integridade e, ou, longevidade; 69,6\% não interferiam, de forma direta, na calçada; $32,8 \%$ das árvores necessitavam de podas por estarem acima da fiação elétrica; $0,5 \%$ necessitava de substituição; e 5,7\% precisavam ser removidas. Com relação à altura total das árvores, 63,0\% apresentavam crescimento menor que $5 \mathrm{~m}$; e $65,3 \%$ com altura da primeira bifurcação do tronco inferior a 1,80 m. O Bairro Primavera destacou-se pelo maior número de árvores em sua urbanização. Em contrapartida, o Jardim Amélia apresentou apenas um indivíduo. Dessa forma, observou-se que cada bairro possuía sua particularidade, e ações de manutenção e enriquecimento, tanto relacionadas ao número de espécies quanto à quantidade de indivíduos, deveriam ser consideradas no planejamento urbano-arbóreo daquele município.
\end{abstract}

Palavras-chave: Arboricultura, Silvicultura urbana e Planejamento urbano.

\section{INVENTORY OF THE TREE PLANTING IN SALTO DE PIRAPORA-SP}

\begin{abstract}
The objective of this study was to conduct an urban tree inventory in 24 neighborhoods of Salto de Pirapora-SP by using a qualitative and quantitative evaluation. Therefore, it was obtained information related to: tree species, location of the crown and trunk, the trunk orientation, plant, root interception of the ride, silvicultural treatments, total height and the first bifurcation, as well as characteristics of road space. Eight hundred and sixty-eight individuals were recorded, of which 679 were cataloged in 71 tree species. The most frequent species was Caesalpinia pluviosa, (13.6\% of the total), followed by Ficus benjamina (10.4\%), Lagerstroemia indica (5.0\%) and Terminalia catappa (4.8\%). The condition of the trunk, $68.8 \%$ of the individual trees showed no problems, $85.7 \%$ of the observed trees have adequate sympodial guidance; few trees had damages related to plant health that may interfere with their integrity and, or, longevity, $69.6 \%$ did not interfere directly on the sidewalk; $32.8 \%$ of the trees needed pruning for being above the electrical wiring; $0.5 \%$ needed replacing and $5.7 \%$ required removal. With respect to the total height of the trees, $63.0 \%$ grew less than $5 \mathrm{~m}$, and $65.3 \%$ present height of the first trunk bifurcation lower than $1.80 \mathrm{~m}$. Bairro Primavera was highlighted
\end{abstract}

\footnotetext{
${ }^{1}$ Recebido em 10.01.2012 aceito para publicação em 04.06.2012.

${ }^{2}$ Graduando em Engenharia Florestal pela Universidade Federal de São Carlos, UFSCAR, Brasil.E-mail:<daniele_benatti@florestal.eng.br>, $<$ francisco_silver@hotmail.com>e <ivanka.ivi@gmail.com>.

${ }^{3}$ Departamento de Ciências Ambientais, Universidade Federal de São Carlos, Campus Sorocaba. E-mail: <kellytonello@ufscar.br> e $<$ jose@ufscar.br $>$.
} 
by the largest number of trees in its urbanization. In contrast, Jardim Amelia had only one individual. Thus, it is observed that each neighborhood has its particularity and maintenance actions and enrichment related to both number of species and number of individuals should be considered in urban planning-tree in the city.

Keywords: Urban trees, Urban forest and Urban planning.

\section{INTRODUÇÃO}

Arborizar é incluir espécies vegetais nos espaços viários com os objetivos de ornamentar o ambiente urbano e regular o ciclo hidrológico do local, bem como beneficiar a população por meio da geração de melhorias na qualidade de vida. Os avanços positivos microclimáticos, que são consequências da redução da poluição, são bastante cotados quando se tem um projeto de arborização de uma área, tendo em vista que a presença das espécies arbóreas, além de realizar o mecanismo de filtragem dos gases atmosféricos, pode ser responsável pelo bem-estar social da população. Silva et al. (2007) apontaram que a arborização urbana é funcionalmente relevante, uma vez que promove não somente os benefícios anteriormente citados, mas também a amenização de ruídos e a proteção ao solo.

Segundo Miller (1997), um bom plano de manejo visa maximizar os benefícios da arborização de ruas e reduzir custos públicos (LEAL et al., 2008). Qualquer manejo de dado recurso começa por um inventário. Os inventários são essenciais para localizar pontos para plantio, identificar necessidades de manejo e localizar riscos relacionados a árvores que estejam necessitando de reparos ou remoção. Outras finalidades podem ser relacionadas ao uso de inventários, como gerar informações a respeito da quantidade e do valor de árvores, para fins de relações públicas ou melhorar a consciência do público e dos políticos acerca do valor da arborização (SMILEY; BAKER, 1988).

Em qualquer atividade que envolva o inventário arbóreo urbano é preciso identificar o porte das árvores implantadas com o intuito de verificar se ele está adequado para a largura do passeio, bem como para o afastamento predial, distância dos indivíduos arbóreos em relação à iluminação pública, aos hidrantes e esquinas (BARBEDO et al., 2005), entre outros. Sabendo dos benefícios promovidos à cidade pela arborização urbana, tem-se o inventário dos indivíduos arbóreos do ambiente urbano, onde se obtêm informações necessárias para a realização do diagnóstico da arborização existente que tem como vertentes servir para o planejamento ou replanejamento da arborização e definir práticas de manejo e monitoramento da área de forma mais adequadas (SILVA et al., 2007). Diante dos tipos de inventários arbóreos urbanos, identificar, quantificar e determinar a qualidade das espécies levantadas são de extrema importância para a prognose de resultados (LIMA, 1993).

Em cidades de pequeno porte geralmente se elaboram censos arbóreos para a quantificação e qualificação das espécies vegetais presentes em vias públicas, uma vez que o tempo de trabalho não é extenso e, portanto, os custos são menores (SILVA et al., 2007).

Diante disso, este trabalho teve como objetivo realizar o inventário arbóreo urbano em 24 bairros de Salto de Pirapora, SP (aproximadamente 50\% da área urbana), por meio do método quali-quantitativo, de forma a fornecer subsídios ao planejamento e manutenção desse setor no município.

\section{METODOLOGIA}

O Município de Salto de Pirapora encontra-se no Sudeste do Estado de São Paulo, a $122 \mathrm{~km}$ da capital. Possui uma área total de $283 \mathrm{~km}^{2}$ aproximadamente, sendo a área urbana de $14,58 \mathrm{~km}^{2}$ (PREFEITURA MUNICIPALDE SALTODEPIRAPORA, 2011). Acidade é banhada pelo rio Pirapora e dispõe de aproximadamente 40.132 habitantes (IBGE, 2011).

O clima da região é classificado por Köppen como transição de $\mathrm{Cwb}$ (temperado chuvoso e quente com verão moderadamente quente) para Cwa (temperado seco e quente com verão quente), com temperatura anual média de aproximadamente $22^{\circ} \mathrm{C}$. A altitude média é de $630 \mathrm{~m}$, e a maior parte da vegetação são pastos naturais ou artificiais e plantações de eucalipto (REDE DAS ÁGUAS, 2011).

\subsection{Inventário arbóreo urbano}

O inventário arbóreo urbano foi realizado ao longo do período de julho de 2010 a janeiro de 2011 pelo método quali-quantitativo do tipo censo, considerando-se os bairros: Jardim Luar, Jardim Conde F. Matarazzo Jr., Jardim Vera Lúcia, Jardim Bela Vista, Vila Santa Isabel, 
Jardim São Paulo, Vila Florianos, Jardim Cachoeira, Jardim Alvorada, Jardim Alexandre, Jardim Amélia, Recanto Cidade Nova, Vila Santa Julieta, Terras de São João, Jardim Avenida, Jardim Madalena, Jardim Daniel Haddad, Jardim Santa Bárbara, Campo Largo, Jardim São Carlos, Jardim Santa Helena, Jardim Primavera, Vila Xavier e Loteamento Santa Maria. Desses, procedeu-se com a avaliação qualitativa dos 13 primeiros bairros, ao passo que os dados quantitativos foram coletados em todos os bairros citados.

Cada indivíduo arbóreo foi identificado quanto à espécie à qual pertenciae, e as informações e o processamento delas foram realizados em formulário específico, constando a data de coleta, nome da rua, bairro, número das casas mais próximas a cada árvore mapeada, lado de fiação, largura aproximada da calçada, interferência dos indivíduos na fiação elétrica, marcação geográfica com GPS das árvores situadas no início e fim de cada rua e demais informações relevantes ao estudo, como se segue:

- Condição da copa: sadia (A), quando aparentava bom estado visual, sem sinais de doenças e ausência ou baixa presença de sinais de deficiência nutricional pelo indivíduo arbóreo; copa oriunda de brotação epicórmica (B); copa portando sinais de amarelecimento e queda considerada anormal de folhas e, ou, em estresse (C) ou, ainda, prejudicada por vandalismo (D).

- Condição de tronco: classificados como íntegros (A), sem sinais de injúrias, portando em bom estado físico e visual e em boas condições fisiológicas; possuindo injúrias (B); tronco oco (C); tronco fendido (D); e tronco anelado (E) ou prejudicado por ação de vandalismo (F), seja pregado, furado ou com quaisquer anormalidades semelhantes.

- Inclinação da árvore: adequada de acordo com a espécie e o ambiente em que se encontra (A) ou inclinada (B).

- Fitossanidade: nesta categoria, atenta-se à observação para a presença de cupins na árvore (A); presença de insetos sugadores (B); presença de ferrugens (C); e presença de parasitas (D).

- Influência do sistema radicular das árvores na via pública: danos severos provocados à calçada, dificultando ou inviabilizando a passagem de transeuntes nas vias (A); presença de danos leves (B), com as vias públicas afetadas parcialmente pelas raízes dos indivíduos arbóreos; calçada em bom estado (C).
- Controle silvicultural: necessidade de remoção das árvores, por motivos como risco direto ou indireto aos munícipes e edificações (A); necessidade de substituição (B) em que árvores inadequadas ou covas vazias podem ser substituídas ou plantadas por espécies mais adequadas conforme o ambiente em que se encontram; necessidade de poda (C), quando as copas interferem na visualização de sinalização de trânsito, atingindo fiação elétrica, podendo gerar transtornos e acidentes nas vias.

Tanto a altura total quanto a altura da primeira bifurcação também foram obtidas. Para a altura total, trabalhou-se com três classes: $<5 \mathrm{~m}$, de 5 a $10 \mathrm{~m}$ e $>10 \mathrm{~m}$. Para a altura da primeira bifurcação, as classes estabelecidas foram: d" $1,80 \mathrm{~m}$ e $>1,80 \mathrm{~m}$.

\section{RESULTADOS}

\subsection{Espécies arbóreas urbanas e frequência}

Nos 24 bairros do Município de Salto de Pirapora, SP, foram amostrados 868 indivíduos, onde 679 foram identificados e distribuídos em 71 espécies arbóreas, 129 indivíduos foram classificados como espécies indeterminadas e 60 como mortas (Tabela 1). As 20 espécies mais frequentes perfizeram $64,6 \%$ do total de indivíduos. A espécie de maior frequência foi a Caesalpinia pluviosa, com 118 indivíduos, representando 13,6\% do total, seguido de Ficus benjamin (10,4\%), Lagerstroemia indica $(5,0 \%)$ e Terminalia catappa $(4,8 \%)$. Foram observadas 26 espécies com apenas um indivíduo, correspondendo a $0,03 \%$ da população amostrada.

\subsection{Avaliação qualitativa}

$\mathrm{Na}$ avaliação qualitativa, os 13 bairros totalizaram 372 indivíduos arbóreos.

A maioria das árvores $(82,8 \%)$ apresentou boas condições de copa (A), enquanto $8,9 \%$ delas apresentaram grande quantidade de ramos e folhas jovens (B). Corresponderam a 8,2\% as árvores com sinais evidentes de estresse (C), como queda anormal de folhas e alta proporção de amarelecimento de folhas. Não foram observados indivíduos caracterizados com sinais de vandalismo (D).

Quanto à condição do tronco, 68,8\% dos indivíduos arbóreos não apresentaram problemas (A); 16,4\% possuíam injúrias (B) e 14,8\% das árvores tiveram

Revista Árvore, Viçosa-MG, v.36, n.5, p.887-894, 2012

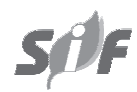


Tabela 1 - Espécies arbóreas identificadas em 24 bairros de Salto de Pirapora, SP.

Table 1 - Tree species identified in 24 neighborhoods in Salto de Pirapora, SP.

\begin{tabular}{|c|c|c|c|}
\hline Nome científico & Nome vulgar & $\mathrm{N}^{\mathrm{o}}$ de indivíduos & Frequência $(\%)$ \\
\hline Annona squamosa & Fruta- do-conde & 1 & 0,1 \\
\hline Bauhinia forficata Link & Pata-de-vaca & 1 & 0,1 \\
\hline Bauhinia variegata & Pata-de-vaca & 23 & 2,6 \\
\hline Bixa orellana & Urucum & 1 & 0,1 \\
\hline Bougainvillea glabra & Primavera & 5 & 0,6 \\
\hline Caesalpinia peltophoroides & Sibipiruna & 10 & 1,2 \\
\hline Caesalpinia pluviosa & Sibipiruna & 118 & 13,6 \\
\hline Callistemon vimindis & Escova-de-garrafa-pendente & 1 & 0,1 \\
\hline Carica papaya & Mamoeiro & 4 & 0,5 \\
\hline Caryota urens & Palmeira-rabo-de-peixe & 1 & 0,1 \\
\hline Cássia fistula $\mathrm{L}$. & Chuva-de-ouro & 4 & 0,5 \\
\hline Celusia fluminensis & Manga-da-praia & 1 & 0,1 \\
\hline Chamaecyparis thyoides & Cipreste-branco & 1 & 0,1 \\
\hline Cinnamodendron dinisii & Pimenteira & 2 & 0,2 \\
\hline Citrus aurantifolia & Limão-galego & 1 & 0,1 \\
\hline Citrus limonium & Limão & 1 & 0,1 \\
\hline Citrus sinensis & Laranja & 1 & 0,1 \\
\hline Codaeum variegatum & Cróton & 6 & 0,7 \\
\hline Copaifera langsdorffii & Copaíba & 2 & 0,2 \\
\hline Cupressus sp. & Cedrinho & 1 & 0,1 \\
\hline Delonix regia & Flamboyant & 12 & 1,4 \\
\hline Dillenia indica & Dilênia & 1 & 0,1 \\
\hline Dracaena marginata & Dracena & 2 & 0,2 \\
\hline Duranta repens aurea & Pingo-de-ouro & 17 & 2,0 \\
\hline Eriobotrya japonica Lindl & Nêspera & 3 & 0,3 \\
\hline Eugenia repanda & Pitanga & 1 & 0,1 \\
\hline Euterpe oleracea & Palmeira & 4 & 0,5 \\
\hline Ficus benjamin & Figueira-benjamim & 90 & 10,4 \\
\hline Ficus variegata & Ficus & 32 & 3,7 \\
\hline Gochnatia polymorpha & Cambará & 1 & 0,1 \\
\hline Handroanthus avellanedae & Ipê-roxo & 2 & 0,2 \\
\hline Handroanthus chrysotrichus & Ipê-peludo & 6 & 0,7 \\
\hline Handroanthus heptaphyllus & Ipê-roxo-de-sete-folhas & 5 & 0,6 \\
\hline Handroanthus impetiginosus & Ipê-roxo & 1 & 0,1 \\
\hline Handroanthus ochraceus & Ipê-amarelo & 5 & 0,6 \\
\hline Handroanthus spp & Ipê & 17 & 2,0 \\
\hline Hibiscus rosa-sinensis & Hibisco (2) & 3 & 0,3 \\
\hline Hibiscus spp. & Hibisco (1) & 1 & 0,1 \\
\hline Inga sp. & Ingá & 1 & 0,1 \\
\hline Jacaranda mimosifolia & Jacarandá & 2 & 0,2 \\
\hline Lagerstroemia indica $\mathrm{L}$. & Resedá & 43 & 5,0 \\
\hline Leucaena leucocephala & Leucena & 7 & 0,8 \\
\hline Ligustrum lucidum & Ligustro & 29 & 3,3 \\
\hline Malpighia glabra & Acerola & 18 & 2,1 \\
\hline Mangifera indica & Mangueira & 10 & 1,2 \\
\hline Maytenus ilicifolia & Espinheira-santa & 1 & 0,1 \\
\hline Michelia champaca $\mathrm{L}$. & Magnólia-amarela & 11 & 1,3 \\
\hline Morus nigra & Amoreira-negra & 1 & 0,1 \\
\hline Murraya exotica & Falsa-murta & 12 & 1,4 \\
\hline Murraya paniculata & Murta & 14 & 1,6 \\
\hline Myrciaria cauliflora & Jabuticaba & 1 & 0,1 \\
\hline Nerium oleander & Espirradeira & 15 & 1,7 \\
\hline
\end{tabular}

Revista Árvore, Viçosa-MG, v.36, n.5, p.887-894, 2012 
Tabela 1 - Cont.

Table 1 - Cont

\begin{tabular}{|c|c|c|c|}
\hline Nome científico & Nome vulgar & $\mathrm{N}^{\circ}$ de indivíduos & Frequência(\%) \\
\hline Persea americana & Abacateiro & 1 & 0,1 \\
\hline Pinheiro & Pinheiro & 2 & 0,2 \\
\hline Platypodium elegans & Amendoim-do-campo & 1 & 0,1 \\
\hline Plumeria rubra & Jasmim-Manga & 2 & 0,2 \\
\hline Polyscias guilfoylei & Árvore-da-felicidade & 1 & 0,1 \\
\hline Prunus serrulata & Cerejeira-do-japão & 1 & 0,1 \\
\hline Psidium guajava & Goiabeira & 15 & 1,7 \\
\hline Punica granatum & Romã & 2 & 0,2 \\
\hline Rosa odorata spp & Roseira & 1 & 0,1 \\
\hline Salix babylonica & Salgueiro-chorão & 13 & 1,5 \\
\hline Schinus molle L. & Aroeira-salsa & 8 & 0,9 \\
\hline Schinus terebinthifolius & Aroeira-pimenteira & 2 & 0,2 \\
\hline Spathodea campanulata & Tulipeira & 3 & 0,3 \\
\hline Tabebuia pentaphilla & Ipê-rosa & 2 & 0,2 \\
\hline Tecoma stans & Ipê-de-jardim & 20 & 2,3 \\
\hline Terminalia catappa & Chapéu-de-sol & 42 & 4,8 \\
\hline Tibouchina granulosa & Quaresmeira & 4 & 0,5 \\
\hline Tibouchina mutabilis & Manacá-da-serra & 5 & 0,6 \\
\hline Yucca elephantipes & Yuca-elefante & 1 & 0,1 \\
\hline Mortas & - & 60 & 6,9 \\
\hline Indeterminadas & - & 129 & 14,9 \\
\hline 71 espécies & & 868 & 100 \\
\hline
\end{tabular}

problemas como: tronco oco (C), fendido (D), anelado (E) ou prejudicado por vandalismo (F): 1,9\%, 2,4\%, $0,8 \%$ e $9,7 \%$, respectivamente. Dessa forma, a maioria dos indivíduos não possuía problemas no tronco, enquanto cerca de $30 \%$ delas apresentaram anormalidades causadas por fatores naturais, como furo de insetos (classe B), e por fatores antrópicos (classes C, D, E e F).

Quanto à inclinação, 85,7\% das árvores observadas possuíam orientação simpodial adequada, enquanto em $14,3 \%$ do total foi constatada inclinação excessiva.

Poucas árvores apresentaram danos relacionados à fitossanidade que poderiam interferir em sua integridade e, ou, longevidade como a presença de cupins, insetos sugadores, parasitas ou ferrugens. Somente $11 \%$ do total estão inseridos nas classes supracitadas, em que a presença de cupins representou $4,2 \%$ dos problemas de fitossanidade. A presença de insetos sugadores, ferrugens e parasitas foram, respectivamente, 3,5\%, $0,3 \%$ e $3,0 \%$.

Com relação à influência das árvores nos passeios, observou-se que $5,7 \%$ destas geraram severos danos às calçadas (A); 24,7\% das árvores provocaram danos leves à calçada, como rachaduras e quebra (B), e 69,6\% não interferiram de forma direta na calçada, sendo classificadas em "Bom estado" (C).

$\mathrm{Na}$ avaliação do controle silvicultural, observou-se que $32,8 \%$ das árvores amostradas necessitavam de podas por estarem acima da fiação elétrica (C). Aqueles indivíduos situados abaixo da fiação, somente $0,5 \%$ necessitavam de substituição (B) e 5,7\%, de remoção (A).

Com relação à altura total das árvores, verificou-se que $63,0 \%$ apresentavam crescimento menor que $5 \mathrm{~m}$; $35,7 \%$ das árvores encontravam-se com altura entre 5 e $10 \mathrm{~m}$ e apenas $1,3 \%$ apresentavam altura superior a $10 \mathrm{~m}$. Nesses mesmos indivíduos, verificou-se que $65,3 \%$ tinham altura da primeira bifurcação do tronco inferior a $1,80 \mathrm{~m}$ e $34,7 \%, \geq 1,80 \mathrm{~m}$.

\subsection{Características do espaço viário e indivíduos arbóreos por bairro}

Os passeios dos 13 bairros analisados como qualitativos apresentaram variação de 1,0 a 2,0 m de largura. Com o total de 81 indivíduos, o Bairro Primavera foi destaque pelo maior número de árvores em sua

Revista Árvore, Viçosa-MG, v.36, n.5, p.887-894, 2012 
urbanização. Em contrapartida, o Jardim Amélia apresentou apenas um indivíduo (Tabela 2). A Rua André Cruconschi, localizada no Bairro Vila Santa Isabel, apresentou 3,1\% das árvores totais utilizadas na arborização urbana (22 indivíduos - maior representação), já as ruas Valter Antônio Rodrigues (Jardim Luar), Diniz F. Leite, Paulo Haddad (Jardim Conde F. Matarazzo Jr.), Antônio Pires de Campos e Paulo Haddad (Jardim Vera Lúcia), Boa Vista, Trinta de Dezembro e São João (Vila Santa Julieta) não apresentaram indivíduo algum.

\section{DISCUSSÃO}

No planejamento da comunidade arbórea de uma cidade ou região, deve-se favorecer a heterogeneidade de espécies, promovendo a variabilidade intra e interespecífica e a boa distribuição dos exemplares (MILANO; DALCIN, 2000). A fim de minimizar riscos de pragas e doenças, Grey e Deneke (1986) recomendaram que cada espécie utilizada na arborização de ruas esteja no intervalo de 10 a $15 \%$ da população total. Já Milano

Tabela 2 - Distribuição de indivíduos na arborização urbana nos 24 bairros estudados em Salto de Pirapora, SP.

Table 2 - Distribution of individuals in urban forestry in the 24 neighborhoods in Salto de Pirapora, SP.

\begin{tabular}{lc}
\hline \multicolumn{1}{c}{ Bairro } & Indivíduos $(\%)$ \\
\hline Jardim Primavera & 11,6 \\
Terras de São João & 8,6 \\
Jardim Madalena & 7,9 \\
Jardim Cachoeira & 7,4 \\
Jardim Bela Vista & 7,3 \\
Jardim São Carlos & 7,3 \\
Jardim Alvorada & 6,9 \\
Jardim São Paulo & 5,7 \\
Jardim Daniel Haddad & 5,0 \\
Vila Florianos & 4,7 \\
Vila Santa Isabel & 3,9 \\
Jardim Avenida & 3,3 \\
Jardim Santa Barbara & 3,1 \\
Jardim Santa Helena & 2,4 \\
Loteamento Santa Maria & 2,1 \\
Vila Santa Julieta & 2,0 \\
Recanto Cidade Nova & 1,9 \\
Campo Largo & 1,7 \\
Jardim Conde F. Matarazzo Jr. & 1,6 \\
Jardim Alexandre & 1,6 \\
Jardim Luar & 1,4 \\
Vila Xavier & 1,4 \\
Jardim Vera Lúcia & 1,0 \\
Jardim Amélia & 0,1 \\
\hline
\end{tabular}

e Dalcin (2000) admitiram um mínimo de 7 a 10\%. A frequência das espécies nos Bairros de Salto de Pirapora, $\mathrm{SP}$, percorridos neste trabalho (Tabela 1) apontou que nenhuma delas ultrapassou tais recomendações, contudo apenas Caesalpinia pluviosa e Ficus benjamina estiveram no intervalo recomendado por esses autores, ao passo que as demais se encontravam abaixo do que se recomendava.

De acordo com Milano e Dalcin (2000), em termos de biodiversidade, a quantidade das espécies utilizadas na arborização urbana de Salto de Pirapora, SP, apresentou-se expressiva. Esses autores recomendaram o número de 10 a 20 espécies para compor a arborização de uma cidade. Entretanto, a baixa frequência de algumas espécies torna-se fator preocupante, uma vez que é interessante e conveniente que se mantenha um número de espécies representativas de biodiversidade como forma de visar as faces estéticas e paisagísticas do local (DANTAS; SOUZA, 2004).

Algumas restrições foram encontradas na literatura quanto ao uso na arborização urbana das duas espécies mais frequentes deste estudo: Caesalpinia pluviosa e Ficus benjamina. Silva et al. (2008) discutiram a dificuldade de uso de Caesalpinia pluviosa por causarem danos a calçadas e encanamentos, acidentes com fiação elétrica e custos com podas. Para Rocha et al. (2004), Fícus benjamina mostrou-se prejudicial à estrutura viária de Nova Iguaçu, RJ, sendo observados também danos ao calçamento e conflitos com as redes aéreas.

O dano provocado no calçamento urbano pela agressividade das raízes dificulta o tráfego de pessoas. Como constatado neste estudo, um percentual aproximado de $70 \%$ das árvores não interferiram nos passeios. Desse modo, pode-se dizer que há grande fração dos indivíduos que não gera esse tipo de problema, sendo, assim, adequados mediante o planejamento urbano.

No caso da utilização de árvores de grande porte em calçadas estreitas, esta se mostra muito mais prática e eficiente, comparando-se com aquela realizada com arbustos ou árvores de pequeno porte. Contudo, deve-se ressaltar que técnicas adequadas de plantio para que não ocorram problemas causados pelas raízes são essenciais também para a fitossanidade das plantas. Além disso, em trabalho realizado por Aguirre Jr. e Lima (2007), árvores de grande porte como Caesalpinia 
pluviosa podem reduzir a amplitude térmica e melhorar as condições microclimáticas na cidade e, consequentemente, a vida dos cidadãos (SILVA et al., 2008).

Neste estudo, grande parte dos indivíduos arbóreos era de pequeno porte. Mesmo sabendo que as espécies Caesalpinia pluviosa e Terminalia catappa são árvores de médio a grande porte, todos os indivíduos registrados dessas espécies tiveram alturas médias inferiores a $5 \mathrm{~m}$, isto é, 4,3 m e 4,8 m, respectivamente. As outras duas espécies com frequência também expressiva, como Ficus benjamin e Lagerstroemia indica L., também apresentaram alturas médias de 4,0 e 4,5 m, respectivamente. Para Rocha et al. (2004), o baixo porte pode ser justificado pela pouca idade dos indivíduos, deficiência no crescimento ou até mesmo pela opção de realizar plantios com espécies de pequeno porte.

A altura da primeira bifurcação também confere aspecto importante ao plano de arborização. De acordo com Rodolfo Jr. et al. (2008), a altura da primeira bifurcação pode vir a ocasionar problemas para pedestres quando a copa se encontra voltada para o passeio, ou para meio de transportes quando esta se encontra voltada para a rua. Como observado neste estudo, a maioria dos indivíduos arbóreos possuía a altura da primeira bifurcação $<1,80 \mathrm{~m}$. Tal resultado pode ser devido ao plantio de indivíduos jovens na arborização urbana que ainda estavam em fase de crescimento e, nesse sentido, sugere-se que seja realizada uma melhor condução para que não venham apresentar problemas no futuro; ou, ainda, o plantio de indivíduos arbóreos já maduros que necessitam de tratos silviculturais.

A inclinação dos indivíduos também pode demonstrar em longo prazo perigo aos moradores e transeuntes nas vias, com risco de queda da árvore sobre residências ou construções e queda de ramos corroborando com uma estética prejudicial às vias públicas. Contudo, grande percentual dos indivíduos arbóreos dos bairros percorridos apresentou-se adequados neste quesito.

Verificou-se a necessidade da realização de práticas silviculturais como a poda, a fim de atenuar os conflitos com a fiação de rede elétrica e postes de iluminação. O planejamento inadequado das espécies para determinados locais nas vias pode vir a potencializar esses conflitos, e o crescimento incontido da copa pode interferir negativamente, ocasionando perturbações aos munícipes e, consequentemente, aumentando um sentimento de aversão à presença de arborização urbana. Christo e Dias (2007) recomendaram, ainda, a realização de podas por técnicos especializados como forma de reduzir lesões nas árvores e, sobretudo, focar o desenvolvimento regular dessas espécies.

Com relação à arborização, observou-se que os 24 bairros diferiam entre si quanto ao planejamento da arborização urbana (Tabela 2), com grande variação na distribuição de indivíduos arbóreos, o que indica a necessidade de readequação nesse sentido. Estudo semelhante foi desenvolvido por Rocha et al. (2004), que observaram que alguns bairros do Município de Nova Iguaçu, RJ, possuíam poucos indivíduos arbóreos e, ou, se encontravam muito distantes, evidenciando a má qualidade da arborização dos bairros.

\section{CONCLUSÃO}

O inventário realizado neste estudo contribuirá para futuras ações da silvicultura urbana no Município de Salto de Pirapora, SP. Ao descrever a quantidade e qualidade das árvores encontradas na área urbana, o estudo reforça a valorização da arborização ali existente e destaca a necessidade de algumas intervenções para melhoria do ambiente.

A distribuição de indivíduos arbóreos entre os bairros foi considerada inadequada, indicando maior necessidade de planejamento na implantação da arborização urbana com o incremento por espécies adequadas a essas condições. São necessárias, ainda, medidas atenuadoras e restauradoras na tentativa de promover a melhoria da cidade como um todo, incluindo, principalmente, a educação ambiental por parte dos moradores, visando à conservação dos recursos naturais do município.

\section{AGRADECIMENTOS}

À Prefeitura Municipal de Salto de Pirapora, pelo apoio e auxílio na obtenção das informações essenciais ao desenvolvimento deste trabalho; e à PróReitoria de Extensão da UFSCar, pelo apoio financeiro.

\section{REFERÊNCIAS}

\author{
AGUIRRE JR, J.; LIMA, A. M. L. P. Uso de \\ árvores $\mathrm{e}$ arbustos em cidades \\ brasileiras. Revista da Sociedade \\ Brasileira de Arborização Urbana, \\ Piracicaba, v.2, n.4, p. 50-66, 2007.
}


BARBEDO, A. S. C. et.al. Manual técnico de arborização urbana. 2.ed. São Paulo: PMSPSVMA, 2005. 45p.

CHRISTO, J. A.; DIAS, A. N. Inventário florestal da arborização urbana do centro da cidade de Prudentópolis -PR. Revista Eletrônica Lato Sensu, Prudentópolis, n. 1, p. 76-93, 2007.

DANTAS, C. I.; SOUZA, C. M. C. Arborização urbana na cidade de Campina Grande - PB: inventário e suas espécies. Revista de Biologia e Ciências da Terra, v. 4, n. 2, s.p., 2004.

GREY, G. W., DENEKE, F. J. Urban forestry. New York, John Wiley \& Sons, 1986. 279 p.

IBGE, Censo Demográfico 2010. Disponível em: http://www.censo2010.ibge.gov.br/sinopse/ index.php?uf=35\&dados=1. Acesso em 10/06/2011

LEAL, L.; BIONDI, D.; ROCHADELLI, R. Custos de implantação e manutenção da arborização de ruas da cidade de Curitiba, PR. Rev. Árvore, Viçosa, v. 32, n. 3, p. 547-555, 2008.

LIMA, A.M. L. P. de.: Análise da arborização viária na área central e seu entorno. Piracicaba. 1993. 238p. Tese (Doutorado em Solos e Nutrição de Plantas) Escola Superior de Agricultura "Luiz de Queiroz". Universidade de São Paulo.

Milano, M.; DAlCiN, E. Arborização de vias públicas. Rio de Janeiro: LIGHT, 2000. 226p.
MILLER, R. W. Street tree inventories, 6. In: MILlER, R. W. Urban Forestry: Planning and Managing Urban Greenspaces. 2.ed. New Jersey: Prentice Hall, 1997. 502p.

REDE DAS ÁGUAS. Caracterização Geral da URGHI 10. Disponível em <http:// www.rededasaguas.org.br/comite/relsmtseg.pdf $>$. Acesso em 22 de jun. 2011.

ROCHA, R.T.; LELES, P.S.S.; OLIVEIRA NETO, S. Arborização de vias públicas em Nova Iguaçu, RJ: o caso dos bairros rancho novo e centro. Rev. Árvore, Viçosa, MG, v.28, n.4, p.599-607, 2004.

RODOLFO JR. et al. Análise da arborização urbana em bairros da cidade de Pombal no Estado da Paraíba. Revista da Sociedade Brasileira de Arborização Urbana, Piracicaba - SP, v.3, n.4, p.3-19, 2008.

SALTO DE PIRAPORA. Prefeitura Municipal. NOSSA HISTÓRIA. Disponível em <http:// www.saltodepirapora.sp.gov.br/>. Acesso em 22de jun. de 2011 de jun. de 2011.

SILVA, A. G.; PAIVA H. N.; GONÇALVES, W. Avaliando a arborização urbana. Viçosa: Aprenda Fácil, 2007. 346p.

SILVA, L.F. et al. Interceptação da chuva pelas copas das espécies de Caesalpinia pluviosa DC. (Sibipiruna) e Tipuana tipu O. Kuntze (Tipuana) em arborização urbana. Scientia Forestalis, Piracicaba, v. 36, n. 80, p. 307-315, 2008.

SMILEY, E.T.; BAKER, F.A. Options in Street Tree Inventories. Journal of Aboriculture, 14(2), p. 36-42, 1988. 